This item was submitted to Loughborough's Research Repository by the author.

Items in Figshare are protected by copyright, with all rights reserved, unless otherwise indicated.

\title{
The half-levels of the FO2 alternation hierarchy
}

\section{PLEASE CITE THE PUBLISHED VERSION}

https://doi.org/10.1007/s00224-016-9712-2

\section{PUBLISHER}

(C) Springer

\section{VERSION}

AM (Accepted Manuscript)

\section{PUBLISHER STATEMENT}

This work is made available according to the conditions of the Creative Commons Attribution-NonCommercialNoDerivatives 4.0 International (CC BY-NC-ND 4.0) licence. Full details of this licence are available at: https://creativecommons.org/licenses/by-nc-nd/4.0/

\section{LICENCE}

CC BY-NC-ND 4.0

\section{REPOSITORY RECORD}

Fleischer, Lukas, Manfred Kufleitner, and Alexander Lauser. 2019. "The Half-levels of the FO2 Alternation Hierarchy". figshare. https://hdl.handle.net/2134/31948. 


\title{
The half-levels of the $\mathrm{FO}^{2}$ alternation hierarchy
}

\author{
Lukas Fleischer Manfred Kufleitner* Alexander Lauser \\ University of Stuttgart, $\mathrm{FMI}^{\dagger}$
}

\begin{abstract}
The alternation hierarchy in two-variable first-order logic $\mathrm{FO}^{2}[<]$ over words was shown to be decidable by Kufleitner and Weil, and independently by Krebs and Straubing. We consider a similar hierarchy, reminiscent of the half levels of the dot-depth hierarchy or the Straubing-Therien hierarchy. The fragment $\Sigma_{m}^{2}$ of $\mathrm{FO}^{2}$ is defined by disallowing universal quantifiers and having at most $m-1$ nested negations. The Boolean closure of $\Sigma_{m}^{2}$ yields the $m^{\text {th }}$ level of the $\mathrm{FO}^{2}$-alternation hierarchy. We give an effective characterization of $\Sigma_{m}^{2}$, i.e., for every integer $m$ one can decide whether a given regular language is definable in $\Sigma_{m}^{2}$. Among other techniques, the proof relies on an extension of block products to ordered monoids.
\end{abstract}

Keywords: regular language; finite monoid; positive variety; first-order logic

\section{Introduction}

The study of logical fragments over words has a long tradition in computer science. Its starting point was the seminal Büchi-Elgot-Trakhtenbrot Theorem from the early 1960s stating that a language is regular if and only if it is definable in monadic second-order logic [2, 7, 41]. A decade later, in 1971, McNaughton and Papert showed that a language is definable in first-order logic if and only if it is star-free [19]. Combining this result with Schützenberger's famous characterization of the star-free languages in terms of finite aperiodic monoids [27] shows that it is decidable whether a given regular language is first-order definable. Since then, many logical fragments were investigated, see e.g. [4, 34] for overviews.

The motivation for such results is two-fold. First, restricted fragments often yield more efficient algorithms for computational problems such as satisfiability or separability. Second, logical fragments give rise to a descriptive complexity: The simpler the fragment to define a language, the simpler the language. This approach is helpful in understanding the rich structure of regular languages.

\footnotetext{
${ }^{*}$ Supported by the German Research Foundation (DFG) under grant DI 435/5-2.

Email: kufleitner@fmi.uni-stuttgart.de

${ }^{\dagger}$ This is the revised full version of a conference paper at CSR 2014 [8].
} 
Logical fragments are usually defined by restricting some resources in formulas. The three most natural restrictions are the quantifier depth (i.e., the number of nested quantifiers), the alternation depth (i.e., the number alternations between existential and universal quantification), and the number of variables. With respect to deciding definability, quantifier depth is not very interesting since for any fixed quantifier depth only finitely many languages are definable (which immediately yields decidability), see e.g. [5]. The situation with alternation in first-order logic is totally different: Only the first level [11, 29] (i.e., no alternation) and the second level [24, 33] are known to be decidable. By a result of Thomas [40] the alternation hierarchy in first-order logic is tightly connected with the dot-depth hierarchy [3] or the Straubing-Thérien hierarchy [32, 37], depending on the presence or absence of the successor predicate. Some progress in the study of the dot-depth hierarchy and the Straubing-Thérien hierarchy was achieved by considering the half-levels. For example, the levels $1 / 2,3 / 2$, and $5 / 2$ in each of the two hierarchies are decidable $[9,20,21,23,24]$. The half levels also have a counterpart in the alternation hierarchy of first-order logic by requiring existential quantifiers in the first block. Another point of view on the half levels is to disallow universal quantifiers and to restrict the number of nested negations.

Regarding the number of variables, Kamp showed that linear temporal logic is expressively complete for first-order logic over words [10]. Since every modality in linear temporal logic can be defined using three variables, first-order logic with only three different names for the variables (denoted by $\mathrm{FO}^{3}$ ) defines the same languages as full first-order logic. This result is often stated as $\mathrm{FO}^{3}=\mathrm{FO}$. Allowing only two variable names yields the proper fragment $\mathrm{FO}^{2}$ of first-order logic. Thérien and Wilke [39] showed that a language is $\mathrm{FO}^{2}$ definable if and only if its syntactic monoid belongs to the variety DA and, since the latter is decidable, one can effectively check whether a given regular language is $\mathrm{FO}^{2}$-definable. For further information on the numerous characterizations of $\mathrm{FO}^{2}$ we refer to [4, 36].

Inside $\mathrm{FO}^{2}$, the alternation depth is also a natural restriction. One difference to full first-order logic is that one cannot rely on prenex normal forms as a simple way of defining the alternation depth. Weil and the second author gave an effective algebraic characterization of the $m^{\text {th }}$ level $\mathrm{FO}_{m}^{2}$ of this hierarchy. More precisely, they showed that it is possible to ascend the $\mathrm{FO}^{2}$-alternation hierarchy using so-called Mal'cev products [18], which in this particular case preserve decidability. There are two main ingredients in the proof. The first one is a combinatorial tool known as rankers [43] or turtle programs [28], and the second is a relativization property of two-variable first-order logic. These two ingredients are then combined using a proof method introduced in [13. Krebs and Straubing gave another effective characterization of $\mathrm{FO}_{m}^{2}$ in terms of identities of $\omega$-terms using completely different techniques [12, 35]; their proof relies on so-called block products.

In this paper we consider the half-levels $\Sigma_{m}^{2}$ of the $\mathrm{FO}^{2}$-alternation hierarchy. A language is definable in $\Sigma_{m}^{2}$ if it is definable in $\mathrm{FO}^{2}$ without universal quantifiers and with at most $m-1$ nested negations. One can also think of $\Sigma_{m}^{2}$ as those $\mathrm{FO}^{2}$-formulas which on every path of their parse tree have at most $m$ blocks of quantifiers, with the outermost block being existential. The main contribution of this paper are $\omega$-terms $U_{m}$ and $V_{m}$ such that an $\mathrm{FO}^{2}$-definable language is $\Sigma_{m}^{2}$-definable if and only if its ordered 
syntactic monoid satisfies $U_{m} \leqslant V_{m}$. For a given regular language it is therefore decidable whether it is definable in $\Sigma_{m}^{2}$ by first checking whether it is $\mathrm{FO}^{2}$-definable and if so, then verifying whether $U_{m} \leqslant V_{m}$ holds in its ordered syntactic monoid. Moreover, for every $\mathrm{FO}^{2}$-definable language $L$ one can compute the smallest integer $m$ such that $L$ is definable in $\Sigma_{m}^{2}$.

The proof step from the identities to logic is a refinement of the approach of Weil and the second author [18] which in turn uses a technique from [13, Section IV]. While the proof method in [13] is quite general and can be applied for solving various other problems [14, 15, 16, 17], it relies on closure under negation. A very specific modification is necessary in order to get the scheme working in the current situation.

The proof for showing that $\Sigma_{m}^{2}$ satisfies the identity $U_{m} \leqslant V_{m}$ is an adaptation of Straubing's proof [35] to ordered monoids. Straubing's proof relies on two-sided semidirect products and the block product principle. As a preparation, we extend both tools to the situation where the first factor is an ordered monoid. In the case of one-sided semidirect products, Pin and Weil used ordered alphabets for allowing ordered monoids on both sides [23]. Even though we conjecture that ordered alphabets should also work for two-sided semidirect products, we do not depend on this more general setting.

\section{Preliminaries}

The free monoid over the alphabet $A$ is denoted by $A^{*}$. Its neutral element is the empty word $\varepsilon$. Let $u=a_{1} \cdots a_{k}$ with $a_{i} \in A$ be a finite word. The alphabet (also known as the content) of $u$ is $\operatorname{alph}(u)=\left\{a_{1}, \ldots, a_{k}\right\}$, its length is $|u|=k$, and the positions of $u$ are $1, \ldots, k$. We say that $i$ is an a-position of $u$ if $a_{i}=a$. The word $u$ is a (scattered) subword of $w$ if $w \in A^{*} a_{1} \cdots A^{*} a_{k} A^{*}$.

First-order logic. We consider first-order logic $\mathrm{FO}=\mathrm{FO}[<]$ over finite words. The syntax of FO-formulas is

$$
\varphi::=\top|\perp| \lambda(x)=a|x=y| x<y|\neg \varphi| \varphi \vee \varphi|\varphi \wedge \varphi| \exists x \varphi
$$

where $a \in A$ is a letter, and $x$ and $y$ are variables. We consider universal quantifiers $\forall x \varphi$ as an abbreviation of $\neg \exists x \neg \varphi$, and $x \leqslant y$ is a shortcut for $(x=y) \vee(x<y)$. The atomic formulas $T$ and $\perp$ are true and false, respectively. Variables are interpreted as positions of a word, and $\lambda(x)=a$ is true if $x$ is an $a$-position. The semantics of the other constructs is as usual; in particular, $\exists x \varphi$ means that there exists a position $x$ which makes $\varphi$ true, and $x<y$ means that position $x$ is strictly smaller than position $y$. We write $\varphi\left(x_{1}, \ldots, x_{\ell}\right)$ for a formula $\varphi$ if at most the variables $x_{i}$ appear freely in $\varphi$; and we write $u, p_{1}, \ldots, p_{\ell}=\varphi\left(x_{1}, \ldots, x_{\ell}\right)$ if $\varphi$ is true over $u$ when every $x_{i}$ is interpreted as the position $p_{i}$ of $u$. A sentence is a formula without free variables. A first-order sentence $\varphi$ defines the language $L(\varphi)=\left\{u \in A^{*}|u|=\varphi\right\}$, and a language is definable in a first-order fragment $\mathcal{F}$ if it is defined by some sentence in $\mathcal{F}$. 
The formulas $\varphi_{m}$ in the $m^{\text {th }}$ level $\Sigma_{m}$ of the negation nesting hierarchy in FO are defined as follows:

$$
\begin{aligned}
\varphi_{0} & :=\top|\perp| \lambda(x)=a|x=y| x<y\left|\neg \varphi_{0}\right| \varphi_{0} \vee \varphi_{0} \mid \varphi_{0} \wedge \varphi_{0} \\
\varphi_{m} & :=\varphi_{m-1}\left|\neg \varphi_{m-1}\right| \varphi_{m} \vee \varphi_{m}\left|\varphi_{m} \wedge \varphi_{m}\right| \exists x \varphi_{m}
\end{aligned}
$$

This means, for $m \geqslant 1$ the formulas in $\Sigma_{m}$ have at most $m-1$ nested negations over quantifier-free formulas $\varphi_{0}$. Using De Morgan's laws and the following equivalences, one can avoid negations in the quantifier-free formulas $\Sigma_{0}$ if the alphabet $A$ is fixed:

$$
\begin{aligned}
\lambda(x) \neq a & \equiv \bigvee_{b \in A \backslash\{a\}} \lambda(x)=b \\
x \neq y & \equiv(x<y) \vee(y<x) \\
\neg(x<y) & \equiv(x=y) \vee(y<x)
\end{aligned}
$$

Also note that, up to logical equivalence, our definition of $\Sigma_{m}$ coincides with the more common definition in terms of formulas in prenex normal form with at most $m$ blocks of quantifiers which start with an existential block. This can be seen by the usual procedure of renaming the variables and successively moving quantifiers outwards.

The two-variable fragment $\mathrm{FO}^{2}$ of first-order logic uses (and reuses) only two different variables, say $x$ and $y$. Combining $\mathrm{FO}^{2}$ and $\Sigma_{m}$ yields the fragment $\Sigma_{m}^{2}$. That is, we have $\varphi \in \Sigma_{m}^{2}$ if both $\varphi \in \Sigma_{m}$ and $\varphi \in \mathrm{FO}^{2}$. In particular, in this paper the exponent 2 in $\Sigma_{m}^{2}$ is two variables and not for second-order logic. The Boolean closure of $\Sigma_{m}^{2}$ is the $m^{\text {th }}$ level $\mathrm{FO}_{m}^{2}$ of the alternation hierarchy within $\mathrm{FO}^{2}$.

Ordered monoids. Green's relations are an important tool in the study of finite monoids. For $x, y \in M$ let $x \leqslant_{\mathcal{R}} y$ if $x M \subseteq y M$, and let $x \leqslant_{\mathcal{L}} y$ if $M x \subseteq M y$. We write $x \mathcal{R} y$ if both $x \leqslant_{\mathcal{R}} y$ and $y \leqslant_{\mathcal{R}} x$; and we set $x<_{\mathcal{R}} y$ if $x \leqslant_{\mathcal{R}} y$ but not $x \mathcal{R} y$. The relations $\mathcal{L}$ and $<_{\mathcal{L}}$ are defined similarly. An element $x \in M$ is idempotent if $x^{2}=x$. For every finite monoid $M$ there exists an integer $\omega_{M} \geqslant 1$ such that $x^{\omega_{M}}$ is the unique idempotent power generated by $x \in M$. If the reference to $M$ is clear from the context, we simply write $\omega$ instead of $\omega_{M}$.

An ordered monoid $(M, \leqslant)$ is a monoid $M$ equipped with a partial order $\leqslant$ which is compatible with multiplication in $M$; that is, $x \leqslant x^{\prime}$ and $y \leqslant y^{\prime}$ implies $x y \leqslant x^{\prime} y^{\prime}$. Every monoid can be considered as an ordered monoid by using the identity relation as order. If no ambiguity arises, we subsequently use the notation $M$ without explicitly mentioning the order. An order ideal of $M$ is a subset $I \subseteq M$ such that $y \leqslant x$ and $x \in I$ implies $y \in I$. The order ideal generated by a subset $P \subseteq M$ is $\downarrow P=\{x \in M \mid \exists y \in P: x \leqslant y\}$.

A monotone homomorphism $h: M \rightarrow N$ is a monoid homomorphism of ordered monoids $M$ and $N$ such that $x \leqslant y$ implies $h(x) \leqslant h(y)$. Submonoids of ordered monoids naturally inherit the order. A monoid $N$ divides a monoid $M$ if there exists a surjective homomorphism from a submonoid of $M$ onto $N$; moreover, if $M$ and $N$ are ordered, then we require the homomorphism to be monotone. The direct product of ordered monoids $M_{1}, \ldots, M_{k}$ is the usual direct product $M_{1} \times \cdots \times M_{k}$ equipped with the product order, i.e., $\left(x_{1}, \ldots, x_{k}\right) \leqslant\left(y_{1}, \ldots, y_{k}\right)$ if $x_{i} \leqslant y_{i}$ for all $i \in\{1, \ldots, k\}$. The empty direct product is the trivial monoid. 
Varieties and identities. A variety (respectively, positive variety) is a class of finite monoids (respectively, finite ordered monoids) closed under division and finite direct products. By abuse of notation, we sometimes say that an ordered monoid $(M, \leqslant)$ belongs to a variety $\mathbf{V}$ of unordered monoids if $M \in \mathbf{V}$. Both varieties and positive varieties are often defined by identities of $\omega$-terms. We only describe the formal setting for positive varieties. The $\omega$-terms over the variables $X$ are defined inductively: The constant 1 for the neutral element is an $\omega$-term (we always assume $1 \notin X$ ), and every variable $x \in X$ is an $\omega$-term. If $u$ and $v$ are $\omega$-terms, then so are $u v$ and $u^{\omega}$. Here, $\omega$ is considered as a unary operation instead of a fixed integer. Every mapping $h: X \rightarrow M$ to a finite monoid $M$ uniquely extends to $\omega$-terms by setting $h(1)=1, h(u v)=h(u) h(v)$ and $h\left(u^{\omega}\right)=h(u)^{\omega_{M}}$. An ordered monoid $M$ satisfies the identity $U \leqslant V$ for $\omega$-terms $U$ and $V$ if $h(U) \leqslant h(V)$ for all mappings $h: X \rightarrow M$. It satisfies $U=V$ if it satisfies both $U \leqslant V$ and $V \leqslant U$. Every class of ordered monoids defined by a set of identities of $\omega$-terms forms a positive variety. In this paper, we need the following varieties:

- The positive variety $\mathbf{J}^{+}$is defined by the identity $x \leqslant 1$. There is a language theoretic characterization similar to Simon's Theorem in terms of so-called shuffle ideals [20].

- The variety $\mathbf{J}$ is the class of all so-called $\mathcal{J}$-trivial finite monoids. There are several well-known characterizations of this class, the most popular being Simon's Theorem on piecewise testable languages [29]. One can define $\mathbf{J}$ by the identities $(x y z)^{\omega} y=(x y z)^{\omega}=y(x y z)^{\omega}$.

- The variety DA is defined by $(x y z)^{\omega} y(x y z)^{\omega}=(x y z)^{\omega}$. An important property of DA is the following: Suppose $M \in \mathbf{D A}$ and let $u, v, a \in M$. If $v \mathcal{R} u \mathcal{R} u a$, then $v \mathcal{R} v a$; and symmetrically, if $v \mathcal{L} u \mathcal{L} a u$, then $v \mathcal{L} a v$, see e.g. [15, Lemma 1].

Languages and syntactic monoids. A language $L \subseteq A^{*}$ is recognized by a homomorphism $h: A^{*} \rightarrow M$ to an ordered monoid $M$ if $L=h^{-1}(I)$ for some order ideal $I$ of $M$. An ordered monoid $M$ recognizes a language $L \subseteq A^{*}$ if there exists a homomorphism $h: A^{*} \rightarrow M$ which recognizes $L$. The syntactic preorder $\leqslant_{L}$ on words is defined as follows: We set $u \leqslant{ }_{L} v$ for $u, v \in A^{*}$ if $p v q \in L$ implies $p u q \in L$ for all $p, q \in A^{*}$. We write $u \equiv_{L} v$ if both $u \leqslant_{L} v$ and $v \leqslant_{L} u$. The syntactic monoid $M_{L}$ of $L$ is the quotient $A^{*} / \equiv_{L}$ consisting of the equivalence classes of $\equiv_{L}$; it is the unique minimal recognizer of $L$ and it is effectively computable from any reasonable presentation of a given regular language. The syntactic preorder induces a partial order on the $\equiv_{L^{-}}$classes such that $M_{L}$ becomes an ordered monoid. The syntactic homomorphism $h_{L}: A^{*} \rightarrow M_{L}$ is the natural quotient map. The above varieties also have characterizations in terms of logic fragments, see [4] for an overview:

- A language is definable in $\Sigma_{1}$ if and only if it is recognized by a monoid in $\mathbf{J}^{+}$.

- A language is definable in the Boolean closure of $\Sigma_{1}$ if and only if it is recognized by a monoid in $\mathbf{J}$.

- A language is definable in $\mathrm{FO}^{2}$ if and only if it is recognized by a monoid in DA. 


\section{Two-Sided Semidirect Products of Ordered Monoids}

One-sided semidirect products are a well-known construction in both group theory and semigroup theory. Wreath products can be thought of as the most general semidirect product (in the sense that every wreath product can be written as a semidirect product, and every semidirect product of $M$ and $N$ divides the wreath product of $M$ and $N$ ), see e.g. Eilenberg's treatise [6] for details. Pin and Weil generalized one-sided semidirect products and wreath products to ordered monoids [22]. As a more symmetric construction, Rhodes and Tilson introduced two-sided semidirect products [25], see also [26]. In this section, we combine the two generalizations "ordered" and "two-sided" by allowing the first of the two monoids to be ordered. Even though this generalization is straightforward, it is quite important for the (first half of the) proof of our main result.

Let $M$ be an ordered monoid and let $N$ be a monoid. Following [6], we write the operation in $M$ additively to improve readability, but this does not mean that $M$ is commutative. A left action of $N$ on $M$ is a mapping $(n, m) \mapsto n \cdot m$ from $N \times M$ to $M$ such that for all $m, m_{1}, m_{2} \in M$ and all $n, n_{1}, n_{2} \in N$ the following axioms hold:

$$
\begin{aligned}
n \cdot\left(m_{1}+m_{2}\right) & =n \cdot m_{1}+n \cdot m_{2}, \\
\left(n_{1} n_{2}\right) \cdot m & =n_{1} \cdot\left(n_{2} \cdot m\right), \\
1 \cdot m & =m, \\
n \cdot 0 & =0, \\
n \cdot m_{1} & \leqslant n \cdot m_{2} \text { whenever } m_{1} \leqslant m_{2} .
\end{aligned}
$$

To shorten notation, we usually write $n m$ instead of $n \cdot m$. A right action of $N$ on $M$ is defined symmetrically. A left and a right action are compatible if $\left(n_{1} m\right) n_{2}=n_{1}\left(m n_{2}\right)$ for all $m \in M$ and all $n_{1}, n_{2} \in N$. For compatible left and right actions of $N$ on $M$ we define the two-sided semidirect product $M * * N$ as the ordered monoid on the set $M \times N$ with the multiplication

$$
\left(m_{1}, n_{1}\right)\left(m_{2}, n_{2}\right)=\left(m_{1} n_{2}+n_{1} m_{2}, n_{1} n_{2}\right),
$$

and the order given by

$$
\left(m_{1}, n_{1}\right) \leqslant\left(m_{2}, n_{2}\right) \text { if and only if } m_{1} \leqslant m_{2} \text { and } n_{1}=n_{2} .
$$

It is straightforward to verify that $M * * N$ indeed is an ordered monoid for each pair of compatible actions. The two-sided semidirect product with left action $(n, m) \mapsto m$ and right action $(m, n) \mapsto m$ yields the direct product of $M$ and $N$. In this sense the two-sided semidirect product generalizes the usual direct product.

We now define the so-called block product as a particular two-sided semidirect product. Let $M^{N \times N}$ be the ordered monoid of all functions from $N \times N$ to the ordered monoid $M$ with componentwise operation. These functions are ordered by $f_{1} \leqslant f_{2}$ if $f_{1}\left(n_{1}, n_{2}\right) \leqslant$ $f_{2}\left(n_{1}, n_{2}\right)$ for all $n_{1}, n_{2} \in N$. One can view $M^{N \times N}$ as the direct product of $|N|^{2}$ copies of $M$. The block product $M \square N$ is the two-sided semidirect product $M^{N \times N} * * N$ induced 
by the following pair of left and right actions. For $f \in M^{N \times N}$ and $n, n_{1}, n_{2} \in N$ let

$$
\begin{aligned}
& (n f)\left(n_{1}, n_{2}\right)=f\left(n_{1}, n n_{2}\right), \\
& (f n)\left(n_{1}, n_{2}\right)=f\left(n_{1} n, n_{2}\right) .
\end{aligned}
$$

By a similar proof as in the unordered case [25], one can easily show the following result.

Lemma 1. Let $M, M^{\prime}$ be ordered monoids and let $N, N^{\prime}$ be monoids. Then the following properties hold:

(a) Both $M$ and $(N,=)$ divide every two-sided semidirect product $M * * N$.

(b) Every two-sided semidirect product $M * * N$ divides $M \square N$.

(c) If $M$ divides $M^{\prime}$ and $N$ divides $N^{\prime}$, then $M \square N$ divides $M^{\prime} \square N^{\prime}$.

Next, we extend the notion of two-sided semidirect products to varieties. For a positive variety $\mathbf{V}$ and a variety $\mathbf{W}$ we let $\mathbf{V} * * \mathbf{W}$ consist of all ordered monoids dividing a two-sided semidirect product $M * * N$ for some $M \in \mathbf{V}$ and $N \in \mathbf{W}$. For two-sided semidirect products $M * * N$ and $M^{\prime} * * N^{\prime}$, we define a new two-sided semidirect product $\left(M \times M^{\prime}\right) * *\left(N \times N^{\prime}\right)$ by the actions

$$
\begin{aligned}
& \left(n, n^{\prime}\right)\left(m, m^{\prime}\right)=\left(n m, n^{\prime} m^{\prime}\right) \\
& \left(m, m^{\prime}\right)\left(n, n^{\prime}\right)=\left(m n, m^{\prime} n^{\prime}\right)
\end{aligned}
$$

for all $m \in M, m^{\prime} \in M^{\prime}, n \in N$, and $n^{\prime} \in N^{\prime}$. An elementary verification shows that this two-sided semidirect product is isomorphic to $(M * * N) \times\left(M^{\prime} * * N^{\prime}\right)$, and $\mathbf{V} * * \mathbf{W}$ thus forms a positive variety. By Lemma 1 we see that $\mathbf{V} * * \mathbf{W}$ is identical to the positive variety generated by all block products $M \square N$ with $M \in \mathbf{V}$ and $N \in \mathbf{W}$.

For a homomorphism $h_{N}: A^{*} \rightarrow N$ we consider the alphabet $A_{N}=N \times A \times N$ and the length-preserving mapping $\sigma_{h_{N}}: A^{*} \rightarrow A_{N}^{*}$ defined by $\sigma_{h_{N}}\left(a_{1} \cdots a_{n}\right)=b_{1} \cdots b_{n}$, where

$$
b_{i}=\left(h_{N}\left(a_{1} \cdots a_{i-1}\right), a_{i}, h_{N}\left(a_{i+1} \cdots a_{n}\right)\right)
$$

for all $i \in\{1, \ldots, n\}$.

Straubing's wreath product principle [30, 31] characterizes the languages recognized by wreath products. Pin and Weil extended this result to ordered monoids [23], and Thérien [38] and Weil [42] generalized it to block products. The latter result is known as the block product principle. The remainder of this section is devoted to an ordered version of the block product principle, thereby combining the "ordered" and the "two-sided" generalizations of the wreath product principle.

Proposition 2. Let $\mathbf{V}$ be a positive variety, let $\mathbf{W}$ be a variety, let $M$ be a finite ordered monoid, and let $h_{M}: A^{*} \rightarrow M$ be a surjective homomorphism. Then the following conditions are equivalent.

(a) $M \in \mathbf{V} * * \mathbf{W}$.

(b) There exists a homomorphism $h_{N}: A^{*} \rightarrow N$ with $N \in \mathbf{W}$ and a homomorphism $h_{K}: A_{N}^{*} \rightarrow K$ with $K \in \mathbf{V}$ such that for all $u, v \in A^{*}$ :

$$
h_{N}(u)=h_{N}(v) \text { and } h_{K}\left(\sigma_{h_{N}}(u)\right) \leqslant h_{K}\left(\sigma_{h_{N}}(v)\right) \text { implies } h_{M}(u) \leqslant h_{M}(v) .
$$


Proof. (a) $\Rightarrow$ (b): By Lemma 1 there exists $K \in \mathbf{V}$ and $N \in \mathbf{W}$ such that $M$ is a divisor of $K \square N$. By the universal property of free monoids we can assume that $M$ is a submonoid of $K \square N$. We can thus read $h_{M}$ as a homomorphism from $A^{*}$ to $K \square N$. Suppose $h_{M}(a)=\left(f_{a}, n_{a}\right)$ for $a \in A$. Then we define $h_{N}: A^{*} \rightarrow N$ by $h_{N}(a)=n_{a}$, and $h_{K}: A_{N}^{*} \rightarrow K^{N \times N}$ is defined by $h_{K}\left(n_{1}, a, n_{2}\right)=n_{1} f_{a} n_{2}$ for $\left(n_{1}, a, n_{2}\right) \in A_{N}$. Consider $u=a_{1} \cdots a_{k}$ with $a_{i} \in A$ and let $\sigma_{h_{N}}(u)=b_{1} \cdots b_{k}$ with $b_{i} \in A_{N}$. Then we have

$$
h_{M}(u)=\left(f_{a_{1}}, n_{a_{1}}\right) \cdots\left(f_{a_{k}}, n_{a_{k}}\right)=\left(t_{1}+\cdots+t_{k}, h_{N}(u)\right),
$$

where the $i^{\text {th }}$ term is $t_{i}=n_{a_{1}} \cdots n_{a_{i-1}} f_{a_{i}} n_{a_{i+1}} \cdots n_{a_{k}}=h_{K}\left(b_{i}\right)$. This yields $h_{M}(u)=$ $\left(h_{K}\left(\sigma_{h_{N}}(u)\right), h_{N}(u)\right)$.

(b) $\Rightarrow$ (a): For each $a \in A$ we define a function $f_{a}: N \times N \rightarrow K$ by $f_{a}\left(n_{1}, n_{2}\right)=$ $h_{K}\left(n_{1}, a, n_{2}\right)$. Let $h: A^{*} \rightarrow K \square N$ be the homomorphism defined by $h(a)=\left(f_{a}, h_{N}(a)\right)$. The assumption yields $h_{M}(u) \leqslant h_{M}(v)$ for all $u, v \in A^{*}$ with $h(u) \leqslant h(v)$. This means that $M$ divides $K \square N$. In particular, $M \in \mathbf{V} * * \mathbf{W}$.

While Proposition 2 gives a characterization of block products in terms of homomorphisms, the following result goes one step further by providing a language characterization.

Proposition 3. Let $\mathbf{V}$ be a positive variety, let $\mathbf{W}$ be a variety, and let $L \subseteq A^{*}$. The following conditions are equivalent.

(a) $L$ is recognized by an ordered monoid in $\mathbf{V} * * \mathbf{W}$.

(b) There exists a homomorphism $h_{N}: A^{*} \rightarrow N$ with $N \in \mathbf{W}$ such that $L$ is a finite union of languages of the form $\sigma_{h_{N}}^{-1}\left(L_{K}\right) \cap L_{N}$ with $L_{K} \subseteq A_{N}^{*}$ being recognized by a monoid in $\mathbf{V}$ and $L_{N} \subseteq A^{*}$ being recognized by $h_{N}$.

Proof. (a) $\Rightarrow$ (b): Suppose $L$ is recognized by the surjective homomorphism $h_{M}: A^{*} \rightarrow M$ with $M \in \mathbf{V} * * \mathbf{W}$. By Proposition 2 there exist homomorphisms $h_{N}: A^{*} \rightarrow N$ and $h_{K}: A_{N}^{*} \rightarrow K$ with $N \in \mathbf{W}$ and $K \in \mathbf{V}$ such that

$$
h_{N}(u)=h_{N}(v) \text { and } h_{K}\left(\sigma_{h_{N}}(u)\right) \leqslant h_{K}\left(\sigma_{h_{N}}(v)\right) \text { implies } h_{M}(u) \leqslant h_{M}(v) .
$$

We define a function $f: A^{*} \rightarrow N \times K$ by $f(u)=\left(h_{N}(u), h_{K}\left(\sigma_{h_{N}}(u)\right)\right.$. Then $f(L)$ is an order ideal of $K \times N$ satisfying $f^{-1}(f(L))=L$. This yields

$$
\bigcup_{(n, k) \in f(L)} f^{-1}(n, \downarrow k)=\bigcup_{(n, k) \in f(L)} f^{-1}(n, k)=f^{-1}(f(L))=L .
$$

Note that $f^{-1}(n, \downarrow k)=\sigma_{h_{N}}^{-1}\left(h_{K}^{-1}(\downarrow k)\right) \cap h_{N}^{-1}(n)$. The claim follows with $L_{K}=h_{K}^{-1}(\downarrow k)$ and $L_{N}=h_{N}^{-1}(n)$.

(b) $\Rightarrow$ (a): Suppose $L$ is a finite union of languages of the form $\sigma_{h_{N}}^{-1}\left(L_{K}\right) \cap L_{N}$ for $h_{N}$, $L_{K}$ and $L_{N}$ as above. Let $K^{\prime}$ be the direct product of the ordered syntactic monoids of all languages $L_{K} \subseteq A_{N}^{*}$ appearing in the union and let $g: A_{N}^{*} \rightarrow K^{\prime}$ be the corresponding natural homomorphism. For every pair $\left(p^{\prime}, q^{\prime}\right) \in N \times N$ we consider the homomorphism $g_{p^{\prime}, q^{\prime}}: A_{N}^{*} \rightarrow K^{\prime}$ defined by $g_{p^{\prime}, q^{\prime}}\left(n_{1}, a, n_{2}\right)=g\left(p^{\prime} n_{1}, a, n_{2} q^{\prime}\right)$ for $\left(n_{1}, a, n_{2}\right) \in A_{N}$. We define $K=K^{\prime N \times N}$ and $h_{K}: A_{N}^{*} \rightarrow K$ by $h_{K}(w)=\left(g_{p^{\prime}, q^{\prime}}(w)\right)_{\left(p^{\prime}, q^{\prime}\right) \in N \times N}$. Consider 
words $u, v \in A^{*}$ with $h_{N}(u)=h_{N}(v)$ and $h_{K}\left(\sigma_{h_{N}}(u)\right) \leqslant h_{K}\left(\sigma_{h_{N}}(v)\right)$ and suppose $p v q \in L$ for $p, q \in A^{*}$. We want to show $p u q \in L$ which then yields $u \leqslant_{L} v$ in the syntactic preorder of $L$. Let $p v q \in \sigma_{h_{N}}^{-1}\left(L_{K}\right) \cap L_{N}$, that is, $\sigma_{h_{N}}(p v q) \in L_{K}$ and $p v q \in L_{N}$. Since $h_{K}\left(\sigma_{h_{N}}(u)\right) \leqslant h_{K}\left(\sigma_{h_{N}}(v)\right)$, we have $g_{h_{N}(p), h_{N}(q)}\left(\sigma_{h_{N}}(u)\right) \leqslant g_{h_{N}(p), h_{N}(q)}\left(\sigma_{h_{N}}(v)\right)$. Together with $h_{N}(u)=h_{N}(v)$ this yields $g\left(\sigma_{h_{N}}(p u q)\right) \leqslant g\left(\sigma_{h_{N}}(p v q)\right)$ and thus $\sigma_{h_{N}}(p u q) \in$ $L_{K}$. Moreover, $h_{N}(p u q)=h_{N}(p v q)$ implies $p u q \in L_{N}$. Using Proposition 2 this shows that the ordered syntactic monoid of $L$ is in $\mathbf{V} * * \mathbf{W}$.

\section{Decidability of Negation Nesting in $\mathrm{FO}^{2}$}

In this section we give two algebraic characterizations of the languages definable in the fragment $\Sigma_{m}^{2}$ of two-variable first-order logic with a restricted number of nested negations. The first description is in terms of (weakly) iterated two-sided semidirect products with $\mathcal{J}$-trivial monoids. For this we define a sequence of positive varieties by

$$
\begin{aligned}
\mathbf{W}_{1} & =\mathbf{J}^{+}, \\
\mathbf{W}_{m} & =\mathbf{W}_{m-1} * * \mathbf{J}
\end{aligned}
$$

for $m>1$. As for the second characterization, we define sequences of $\omega$-terms $U_{m}$ and $V_{m}$ by setting

$$
\begin{array}{ll}
U_{1}=z, & U_{m}=\left(U_{m-1} x_{m}\right)^{\omega} U_{m-1}\left(y_{m} U_{m-1}\right)^{\omega}, \\
V_{1}=1, & V_{m}=\left(U_{m-1} x_{m}\right)^{\omega} V_{m-1}\left(y_{m} U_{m-1}\right)^{\omega},
\end{array}
$$

where $x_{2}, y_{2}, \ldots, x_{m}, y_{m}, z$ are variables.

Theorem 4. Let $L \subseteq A^{*}$ and let $m \geqslant 1$. The following conditions are equivalent:

(a) $L$ is definable in $\Sigma_{m}^{2}$.

(b) The ordered syntactic monoid of $L$ is in $\mathbf{W}_{m}$.

(c) The ordered syntactic monoid of $L$ is in DA and satisfies $U_{m} \leqslant V_{m}$.

Since condition (C) in Theorem 4 is decidable for any given regular language $L$, this immediately yields the following corollary.

Corollary 5. It is decidable whether a given regular language is definable in $\Sigma_{m}^{2}$.

Note that in condition (c) of Theorem 4 one cannot drop the requirement of the syntactic monoid being in DA. For example, the syntactic monoid of $A^{*} \backslash A^{*} a a A^{*}$ over $A=\{a, b\}$ satisfies the identity $U_{m} \leqslant V_{m}$ for all $m \geqslant 2$. It is nonetheless not $\Sigma_{m}^{2}$-definable, because it is not even $\mathrm{FO}^{2}$-definable (and thus its syntactic monoid is not in DA). The remainder of this paper proves Theorem 4 .

\subsection{From logic to block products}

We begin with the direction $(a) \Rightarrow(b)$. The arguments are similar to Straubing's for characterizing $\mathrm{FO}_{m}^{2}$ in terms of unordered two-sided semidirect products [35]. 
Lemma 6. Let $m \geqslant 1$. If $L$ is definable in $\Sigma_{m}^{2}$, then $M_{L} \in \mathbf{W}_{m}$.

Proof. Let $\varphi$ be a sentence in $\Sigma_{m}^{2}$ such that $L=L(\varphi)$. We may assume that quantifier-free subformulas of $\varphi$ do not contain negations.

The proof proceeds by induction on $m$. For the base case $m=1$, the language $L$ is a finite union of languages of the form $A^{*} a_{1} \cdots A^{*} a_{k} A^{*}$ and thus $p q \in L$ implies $p u q \in L$ for all $p, u, q \in A^{*}$. This means that $M_{L}$ satisfies $x \leqslant 1$ and therefore, $M_{L} \in \mathbf{J}^{+}$, see [20].

Let now $m \geqslant 2$. An innermost block of $\varphi$ is a maximal negation-free subformula $\psi(x)$ of $\varphi$. As in the unordered case, one can show that each block is equivalent to a disjunction of formulas of the form

$$
\begin{aligned}
\lambda(x)=a \wedge & \left(\exists y_{1} \cdots \exists y_{r} \bigwedge_{i=1}^{r}\left(y_{i}<x \wedge \lambda\left(y_{i}\right)=a_{i}\right) \wedge \pi\left(y_{1}, \ldots, y_{r}\right)\right) \wedge \\
& \left(\exists z_{1} \cdots \exists z_{s} \bigwedge_{i=1}^{s}\left(z_{i}>x \wedge \lambda\left(z_{i}\right)=a_{i}^{\prime}\right) \wedge \pi^{\prime}\left(z_{1}, \ldots, z_{s}\right)\right),
\end{aligned}
$$

where $\pi$ and $\pi^{\prime}$ are quantifier-free formulas defining an order on their parameters. Hence, each innermost block $\psi(x)$ requires that $x$ is an $a$-position and that certain subwords appear to the left and to the right of position $x$. Let $k$ be the maximum of all $r$ and $s$ occurring in these blocks. By Simon's Theorem [29], there exists an unordered monoid $N \in \mathbf{J}$ and a homomorphism $h_{N}: A^{*} \rightarrow N$ such that $h_{N}(u)=h_{N}(v)$ if and only if $u$ and $v$ agree on subwords of length at most $k$. Now, the aforementioned blocks can be replaced by a disjunction of formulas $\lambda(x)=\left(n, a, n^{\prime}\right)$ with $n, n^{\prime} \in N$ and $a \in A$ to obtain an equivalent formula over the alphabet $A_{N}$.

After replacing each innermost block, the resulting formula $\varphi^{\prime}$ is in $\Sigma_{m-1}^{2}$. By induction, the corresponding language $L\left(\varphi^{\prime}\right)$ is recognized by a monoid $K \in \mathbf{W}_{m-1}$. We have $L=$ $L(\varphi)=\sigma_{h_{N}}\left(L\left(\varphi^{\prime}\right)\right)$ by construction. Proposition 3 yields $M_{L} \in \mathbf{W}_{m-1} * * \mathbf{J}=\mathbf{W}_{m}$.

\subsection{From block products to identities}

We now give a technique which allows to extend identities for a positive variety $\mathbf{V}$ to identities that hold in $\mathbf{V} * * \mathbf{J}$. It generalizes a result due to Straubing [35] (which we recover as an immediate consequence). It is used in Lemma 8 below for showing that the identity $U_{m} \leqslant V_{m}$ holds in $\mathbf{W}_{m}$ and that $\mathbf{W}_{m}$ is contained in $\mathbf{D A}$, i.e., for the direction (c) $\Rightarrow$ (a) in Theorem 4 .

Lemma 7. Let $P, Q$ and $S, T$ be $\omega$-terms such that every variable in $P$ or $Q$ appears in both $S$ and $T$. Let $\mathbf{V}$ be a positive variety such that every ordered monoid in $\mathbf{V}$ satisfies $P \leqslant Q$. Every monoid in $\mathbf{V} * * \mathbf{J}$ satisfies $S^{\omega} P T^{\omega} \leqslant S^{\omega} Q T^{\omega}$.

Proof. Let $K \in \mathbf{V}$ and $N \in \mathbf{J}$ be ordered monoids. Choose $n \geqslant 1$ such that $x^{n}$ is idempotent for each $x$ in $K, N$, or $K * * N$. We successively replace all subterms of the form $\left(x_{1} \cdots x_{k}\right)^{\omega}$ by $\left(x_{1} \cdots x_{k}\right)^{n}$ in both $P$ and $Q$. It is straightforward to see that the evaluation of $P$ or $Q$ in $K, N$, and $K * * N$ is invariant under this modification. We consider instances of the new terms $P$ and $Q$ (which are just words). Let $\ell=|P|$ and 
$\ell^{\prime}=|Q|$, and let $p_{1}, \ldots, p_{\ell}, q_{1}, \ldots, q_{\ell^{\prime}}, s, t \in K * * N$ be such that the following properties hold:

- Both $s$ and $t$ have a factorization in which each $p_{1}, \ldots, p_{\ell}$ appears as a factor.

- Both $s$ and $t$ have a factorization in which each $q_{1}, \ldots, q_{\ell^{\prime}}$ appears as a factor.

- We have $p_{i}=p_{j}$ if $P$ contains the same variables at positions $i$ and $j$.

- We have $q_{i}=q_{j}$ if $Q$ contains the same variables at positions $i$ and $j$.

We show $s^{n} p_{1} \cdots p_{\ell} t^{n} \leqslant s^{n} q_{1} \cdots q_{\ell^{\prime}} t^{n}$ in $K * * N$, thereby proving the claim. For an element $x \in K * * N$, let $\bar{x} \in K$ and $\hat{x} \in N$ be such that $x=(\bar{x}, \hat{x})$. We have

$$
\begin{aligned}
s^{n} p_{1} \cdots p_{\ell} t^{n}= & \sum_{i=1}^{n} \hat{s}^{i-1} \bar{s}\left(\hat{s}^{n-i} \hat{p}_{1} \cdots \hat{p}_{\ell} \hat{t}^{n}\right)+\sum_{i=1}^{\ell} \hat{s}^{n} \hat{p}_{1} \cdots \hat{p}_{i-1} \bar{p}_{i} \hat{p}_{i+1} \cdots \hat{p}_{\ell} \hat{t}^{n}+ \\
& \sum_{i=1}^{n}\left(\hat{s}^{n} \hat{p}_{1} \cdots \hat{p}_{\ell} \hat{t}^{n-i}\right) \bar{t} \hat{t}^{i-1} \\
= & \sum_{i=1}^{n} \hat{s}^{i-1} \bar{s}\left(\hat{s}^{n-i} \hat{t}^{n}\right)+\hat{s}^{n}\left(\sum_{i=1}^{\ell} \bar{p}_{i}\right) \hat{t}^{n}+\sum_{i=1}^{n}\left(\hat{s}^{n} \hat{t}^{n-i}\right) \bar{t} \hat{t}^{i-1} .
\end{aligned}
$$

The first equality is the definition of the two-sided semidirect product, the second equality follows from the so-called absorbing property $(x y z)^{\omega} y=(x y z)^{\omega}=y(x y z)^{\omega}$ of $\mathbf{J}$ and the distributive law of the actions defining $K * * N$. Since $K$ satisfies $P \leqslant Q$, we have $\bar{p}_{1}+\ldots+\bar{p}_{\ell} \leqslant \bar{q}_{1}+\ldots+\bar{q}_{\ell^{\prime}}$. Substituting this for the second sum in the last line of the displayed equation above and performing the backwards calculation yields $s^{n} p_{1} \cdots p_{\ell} t^{n} \leqslant s^{n} q_{1} \cdots q_{\ell^{\prime}} t^{n}$ as desired.

Lemma 8. Let $m \geqslant 1$. If $M \in \mathbf{W}_{m}$, then $M \in \mathbf{D A}$ and $M$ satisfies $U_{m} \leqslant V_{m}$.

Proof. We proceed by induction on $m$. The positive variety $\mathbf{W}_{1}=\mathbf{J}^{+}$is defined by the identity $x \leqslant 1$; i.e., $U_{1} \leqslant V_{1}$. Therefore, $(x y z)^{\omega}=(x y z)^{2 \omega-1} x y z(x y z)^{\omega} \leqslant$ $(x y z)^{\omega} y(x y z)^{\omega} \leqslant(x y z)^{\omega}$ and thus, $\mathbf{W}_{1} \subseteq \mathbf{D A}$.

Let now $m \geqslant 2$. By the induction hypothesis, every monoid in $\mathbf{W}_{m-1}$ is in DA and satisfies $U_{m-1} \leqslant V_{m-1}$. Using Lemma 7 and setting $P=(x y z)^{\omega} y(x y z)^{\omega}$ and $Q=S=$ $T=(x y z)^{\omega}$ we see that all monoids in $\mathbf{W}_{m}=\mathbf{W}_{m-1} * * \mathbf{J}$ satisfy $(x y z)^{\omega} y(x y z)^{\omega} \leqslant(x y z)^{\omega}$. By swapping $P$ and $Q$, we obtain $(x y z)^{\omega} \leqslant(x y z)^{\omega} y(x y z)^{\omega}$. Each monoid in $\mathbf{W}_{m}$ is thus in DA.

Observe that all variables appearing in $V_{m-1}$ also appear in $U_{m-1}$. Hence, setting $P=U_{m-1}, Q=V_{m-1}, S=\left(U_{m-1} x_{m}\right)$, and $T=\left(y_{m} U_{m-1}\right)$ shows that the identity $U_{m} \leqslant V_{m}$ holds in $\mathbf{W}_{m}$.

\subsection{From identities to logic}

We turn to the implication (c) $\Rightarrow$ (a) in Theorem 4, from $U_{m} \leqslant V_{m}$ back to logic $\Sigma_{m}^{2}$. This is the most difficult step. On a high-level perspective, we want to use induction on $m$, then use the identity $U_{m-1} \leqslant V_{m-1}$ to get to $\Sigma_{m-1}^{2}$, and finally lift this back 
to $\Sigma_{m}^{2}$. An important part of this argument is the ability to restrict (or relativize) the interpretation of $\Sigma_{m}^{2}$-formulas to certain factors of the model which are given by first and last occurrences of letters.

In the following we also have to take the quantifier depth of a formula into account, i.e., the maximal number of nested quantifiers. For an integer $n \geqslant 0$ let $\Sigma_{m, n}^{2}$ be the fragment of $\Sigma_{m}^{2}$ of formulas with quantifier depth at most $n$.

Lemma 9. Let $\varphi \in \Sigma_{m, n}^{2}$ for $m, n \geqslant 0$, and let $a \in A$. There exist formulas $\langle\varphi\rangle_{>\mathrm{X} a} \in$ $\Sigma_{m, n+1}^{2}$ and $\langle\varphi\rangle_{<\mathrm{X} a} \in \Sigma_{m+1, n+1}^{2}$ such that for all $u=u_{1} a u_{2}$ with $a \notin \operatorname{alph}\left(u_{1}\right)$ and $i=\left|u_{1} a\right|$ we have:

$$
\begin{aligned}
& u, p, q \models\langle\varphi\rangle_{<\mathrm{X} a} \quad \text { if and only if } u_{1}, p, q=\varphi \text { for all } 1 \leqslant p, q<i, \\
& u, p, q \models\langle\varphi\rangle_{>\mathrm{X} a} \quad \text { if and only if } u_{2}, p-i, q-i \models \varphi \text { for all } i<p, q \leqslant|u| .
\end{aligned}
$$

Proof. Let $\langle\varphi\rangle_{<X a} \equiv \varphi$ if $\varphi$ is an atomic formula. For conjunction and disjunction, and negation we inductively take $\langle\varphi\rangle_{<\mathrm{X} a} \wedge\langle\psi\rangle_{<\mathrm{X} a}$ and $\langle\varphi\rangle_{<\mathrm{X} a} \vee\langle\psi\rangle_{<\mathrm{X} a}$, and $\neg\langle\varphi\rangle_{<\mathrm{X} a}$, respectively. For existential quantification let

$$
\langle\exists x \varphi\rangle_{<\mathrm{X} a} \equiv \exists x\left(\neg(\exists y \leqslant x: \lambda(y)=a) \wedge\langle\varphi\rangle_{<\mathrm{X} a}\right) .
$$

As usual, swapping the variables $x$ and $y$ yields the corresponding constructions for $y$. Atomic formulas and Boolean combinations in the construction of $\langle\varphi\rangle_{>} \times a$ are as above. For the other formula let

$$
\langle\exists x \varphi\rangle_{>\mathrm{X} a} \equiv \exists x\left((\exists y<x: \lambda(y)=a) \wedge\langle\varphi\rangle_{>\mathrm{X} a}\right) .
$$

The notation in the indices of the formulas mean that we restrict to the positions smaller (respectively, greater) than the first $a$-position (the neXt $a$-position, thence $\mathrm{X}_{a}$ ). Of course there are dual formulas $\langle\varphi\rangle_{<\mathrm{Y} b} \in \Sigma_{m, n+1}^{2}$ as well as $\langle\varphi\rangle_{>\mathrm{Y} b} \in \Sigma_{m+1, n+1}^{2}$ for the last $b$-position (i.e., the Yesterday $b$-position). The next lemma handles the case of the first $a$-position lying beyond the last $b$-position.

Lemma 10. Let $\varphi \in \Sigma_{m, n}^{2}$ for $m, n \geqslant 0$, and let $a, b \in A$. There exists a formula $\langle\varphi\rangle_{(\mathrm{Y} b ; \mathrm{X} a)}$ in $\Sigma_{m+1, n+1}^{2}$ such that for all words $u=u_{1} b u_{2} a u_{3}$ with $b \notin \operatorname{alph}\left(u_{2} a u_{3}\right)$ and $a \notin \operatorname{alph}\left(u_{1} b u_{2}\right)$ and for all $\left|u_{1} b\right|<p, q \leqslant\left|u_{1} b u_{2}\right|$ we have:

$$
u, p, q \models\langle\varphi\rangle_{(\mathrm{Y} b ; \times a)} \quad \text { if and only if } u_{2}, p-\left|u_{1} b\right|, q-\left|u_{1} b\right| \models \varphi \text {. }
$$

Proof. Atomic formulas and Boolean combinations are straightforward. Let the macro $\mathrm{Y}_{b}<x<\mathrm{X}_{a}$ stand for $\neg(\exists y \leqslant x: \lambda(y)=a) \wedge \neg(\exists y \geqslant x: \lambda(y)=b)$. Using this shortcut, we set $\langle\exists x \varphi\rangle_{(\mathrm{Y} b ; \mathrm{X} a)} \equiv \exists x\left(\left(\mathrm{Y}_{b}<x<\mathrm{X}_{a}\right) \wedge\langle\varphi\rangle_{(\mathrm{Y} b ; \mathrm{X} a)}\right)$.

Let $h: A^{*} \rightarrow M$ be a homomorphism. The $\mathcal{L}$-factorization of a word $u$ is the unique factorization $u=s_{0} a_{1} \cdots s_{\ell-1} a_{\ell} s_{\ell}$ with $s_{i} \in A^{*}$ and so-called markers $a_{i} \in A$ such that $h\left(s_{\ell}\right) \mathcal{L} 1$ and $h\left(s_{i} a_{i+1} \cdots s_{\ell-1} a_{\ell} s_{\ell}\right)>_{\mathcal{L}} h\left(a_{i} s_{i} \cdots a_{\ell} s_{\ell}\right) \mathcal{L} h\left(s_{i-1} a_{i} \cdots s_{\ell-1} a_{\ell} s_{\ell}\right)$ for all $i$. Note that $\ell<|M|$. Furthermore, if $M \in \mathbf{D A}$, then $a_{i} \notin \operatorname{alph}\left(s_{i}\right)$. Let $D_{\mathcal{L}}(u)$ consist of the positions of the markers, i.e., let $D_{\mathcal{L}}(u)=\left\{\left|s_{0} a_{1} \cdots s_{i-1} a_{i}\right| \mid 1 \leqslant i \leqslant \ell\right\}$. The 
$\mathcal{R}$-factorization and $D_{\mathcal{R}}$ are defined left-right symmetrically. In particular $D_{\mathcal{R}}(u)$ is the set of all positions $|p a|$ of $u$ for prefixes $p a$ of $u$ such that $h(p)>_{\mathcal{R}} h(p a)$ for some $a \in A$. The following lemma combines the $\mathcal{R}$-factorization with the $\mathcal{L}$-factorization for monoids in DA such that, starting with $\Sigma_{m}^{2}$, one can express $\Sigma_{m-1}^{2}$-properties of the factors. To formulate this feature we set $u \leqslant{ }_{m, n}^{\mathrm{FO} 2} v$ for words $u, v \in A^{*}$ if $v \models \varphi$ implies $u \models \varphi$ for all $\varphi \in \Sigma_{m, n}^{2}$.

Lemma 11. Let $h: A^{*} \rightarrow M$ be a homomorphism with $M \in \mathbf{D A}$, let $m \geqslant 2$ and $n \geqslant 0$ be integers, and let $u, v \in A^{*}$ with $u \leqslant_{m, 2|M|+n}^{\mathrm{FO} 2} v$. There exist factorizations $u=s_{0} a_{1} \cdots s_{\ell-1} a_{\ell} s_{\ell}$ and $v=t_{0} a_{1} \cdots t_{\ell-1} a_{\ell} t_{\ell}$ with $a_{i} \in A$ and $s_{i}, t_{i} \in A^{*}$ such that the following properties hold for all $i \in\{1, \ldots, \ell\}$ :

(a) $s_{i} \leqslant{ }_{m-1, n}^{\mathrm{FO} 2} t_{i}$,

(b) $h\left(s_{0}\right) \mathcal{R} 1$ and $h\left(t_{0} a_{1} \cdots t_{i-1} a_{i}\right) \mathcal{R} h\left(t_{0} a_{1} \cdots t_{i-1} a_{i} s_{i}\right)$,

(c) $h\left(s_{\ell}\right) \mathcal{L} 1$ and $h\left(a_{i} s_{i} \cdots a_{\ell} s_{\ell}\right) \mathcal{L} h\left(s_{i-1} a_{i} \cdots a_{\ell} s_{\ell}\right)$.

Proof. Note that in property (b) the suffix is $s_{i}$ and not $t_{i}$. We want to prove the claim by an induction, for which we have to slightly generalize the claim. Apart from the words $u$ and $v$ from the premises of the lemma we also consider an additional word $p$ which serves as a prefix for $v$. The proof is by induction on $\left|D_{\mathcal{R}}(p v) \backslash D_{\mathcal{R}}(p)\right|$. The assumptions are $u \leqslant_{m, n^{\prime}}^{\mathrm{FO} 2} v$, where $n^{\prime}=n+\left|D_{\mathcal{R}}(p v) \backslash D_{\mathcal{R}}(p)\right|+\left|D_{\mathcal{L}}(u)\right|+1$. We shall construct factorizations $u=s_{0} a_{1} \cdots s_{\ell-1} a_{\ell} s_{\ell}$ and $p v=p t_{0} a_{1} \cdots t_{\ell-1} a_{\ell} t_{\ell}$ such that properties (a) and (c) hold, but instead of (b) we have $h\left(p t_{0} a_{1} \cdots t_{i-1} a_{i}\right) \mathcal{R} h\left(p t_{0} a_{1} \cdots t_{i-1} a_{i} s_{i}\right)$ and $h\left(p s_{0}\right) \mathcal{R} h(p)$. We thus recover the lemma using an empty prefix $p$.

Let $u=s_{0}^{\prime} c_{1} \cdots s_{\ell^{\prime}-1}^{\prime} c_{\ell^{\prime}} s_{\ell^{\prime}}^{\prime}$ be the $\mathcal{L}$-factorization (in particular $c_{i} \notin \operatorname{alph}\left(s_{i}^{\prime}\right)$ ) and let $v=t_{0}^{\prime} c_{1} \cdots t_{\ell^{\prime}-1}^{\prime} c_{\ell^{\prime}} t_{\ell^{\prime}}^{\prime}$ where $c_{i} \notin \operatorname{alph}\left(t_{i}^{\prime}\right)$ for all $i$. The factorization of $v$ exists because by assumption $u$ and $v$ agree on subwords of length $\ell^{\prime}$. The dual of Lemma 9 yields $s_{0}^{\prime} c_{1} \cdots c_{\ell^{\prime}-i} s_{\ell^{\prime}-i}^{\prime} \leqslant_{m-1, n^{\prime}-i}^{\mathrm{FO} 2} t_{0}^{\prime} c_{1} \cdots c_{\ell^{\prime}-i} t_{\ell^{\prime}-i}^{\prime}$ as well as $s_{i}^{\prime} \leqslant_{m-1, n}^{\mathrm{FO} 2} t_{i}^{\prime}$ for all $i$.

First suppose $D_{\mathcal{R}}(p)=D_{\mathcal{R}}(p v)$. In this case $h(p) \mathcal{R} h(p v)$, and therefore, $h(p) \mathcal{R} h(p x)$ for all $x \in B^{*}$, where $B=\operatorname{alph}(v)$. So in particular we have that $h\left(p t_{0}^{\prime} c_{1} \cdots t_{i-1}^{\prime} c_{i}\right) \mathcal{R}$ $h\left(p t_{0}^{\prime} c_{1} \cdots t_{i-1}^{\prime} c_{i} s_{i}^{\prime}\right)$ because $\operatorname{alph}(u)=B$. Setting $a_{i}=c_{i}, s_{i}=s_{i}^{\prime}$, and $t_{i}=t_{i}^{\prime}$ yields a factorization with the desired properties.

Suppose now $D_{\mathcal{R}}(p) \subsetneq D_{\mathcal{R}}(p v)$, and let $s$ be the longest prefix of $u$ such that $h(p) \mathcal{R}$ $h(p s)>_{\mathcal{R}} h(p s a)$ for some $a \in A$. Such a prefix exists as $\operatorname{alph}(u)=\operatorname{alph}(v)$. We have $a \notin \operatorname{alph}(s)$ by $M \in \mathbf{D A}$. Let $t$ be the longest prefix of $v$ with $a \notin \operatorname{alph}(t)$. Using Lemma 9 we see $\operatorname{alph}(t) \subseteq \operatorname{alph}(s)$. Let $k$ and $k^{\prime}$ be maximal such that $s_{0}^{\prime} c_{1} \cdots s_{k-1}^{\prime} c_{k}$ is a prefix of $s$ and such that $t_{0}^{\prime} c_{1} \cdots t_{k^{\prime}-1}^{\prime} c_{k^{\prime}}$ is a prefix of $t$. We claim $k=k^{\prime}$. For instance, suppose $k<k^{\prime}$. Then $a c_{k+1} \cdots c_{\ell^{\prime}}$ is a subword of $u$ but not of $v$ (since $c_{k+1} t_{k+1}^{\prime} \cdots c_{\ell^{\prime}} t_{\ell^{\prime}}^{\prime}$ is the shortest suffix of $v$ with the subword $c_{k+1} \cdots c_{\ell^{\prime}}$ and since there is no $a$-position in $\left.t_{0}^{\prime} c_{1} \cdots t_{k}^{\prime}\right)$. Let $a_{i}=c_{i}$ for $i \in\{1, \ldots, k\}$, and let $s_{i}=s_{i}^{\prime}$ and $t_{i}=t_{i}^{\prime}$ for $i \in\{0, \ldots, k-1\}$. Let $s_{k}$ and $t_{k}$ such that $s=s_{0} c_{1} \cdots s_{k-1} c_{k} s_{k}$ and $t=t_{0} c_{1} \cdots t_{k-1} c_{k} t_{k}$. Lemma 10 yields $s_{k} \leqslant m_{m-1, n}^{\mathrm{FO} 2} t_{k}$.

Let $u=s a u^{\prime}$ and $v=t a v^{\prime}$, and let $p^{\prime}=p t a$. For all $i \in\{0, \ldots, k\}$ we have $h\left(p t_{0} a_{1} \cdots t_{i-1} a_{i}\right) \mathcal{R} h\left(p t_{0} a_{1} \cdots t_{i-1} a_{i} s_{i}\right)$ because $\operatorname{alph}(t) \subseteq \operatorname{alph}(s)$. We note that $h\left(a_{i+1} s_{i+1} \cdots a_{k} s_{k} a u^{\prime}\right) \mathcal{L} h\left(s_{i} a_{i+1} s_{i+1} \cdots a_{k} s_{k} a u^{\prime}\right)$. Since $M \in \mathbf{D A}$ we see $h(p)>_{\mathcal{R}} h\left(p^{\prime}\right)$ 
and thus $D_{\mathcal{R}}(p) \subsetneq D_{\mathcal{R}}\left(p^{\prime}\right)$. Using the formulas $\langle\varphi\rangle_{>\mathrm{X} a}$ from Lemma 9 yields $u^{\prime} \leqslant_{m, n^{\prime}-1}^{\mathrm{FO} 2} v^{\prime}$. As $n^{\prime} \geqslant\left|D_{\mathcal{R}}\left(p^{\prime} v^{\prime}\right) \backslash D_{\mathcal{R}}\left(p^{\prime}\right)\right|+\left|D_{\mathcal{L}}\left(u^{\prime}\right)\right|+2$ we can apply induction to obtain factorizations $u^{\prime}=s_{k+1} a_{k+2} \cdots s_{\ell-1} a_{\ell} s_{\ell}$ and $v^{\prime}=t_{k+1} a_{k+2} \cdots t_{\ell-1} a_{\ell} t_{\ell}$. Setting $a_{k+1}=a$ yields the desired factorizations.

The preceding lemma enables induction on the parameter $m$. We want to show that, starting with a homomorphism onto a monoid satisfying $U_{m} \leqslant V_{m}$, preimages of $\leqslant$-order ideals are unions of $\leqslant_{m, n}^{\mathrm{FO}}$-order ideals for sufficiently large $n$. Intuitively, a string rewriting technique yields the largest quotient satisfying the identity $U_{m-1} \leqslant V_{m-1}$. One rewriting step corresponds to one application of the identity $U_{m-1} \leqslant V_{m-1}$ of level $m-1$. Such rewriting steps can be lifted to the identity $U_{m} \leqslant V_{m}$ in the contexts they are applied.

Proposition 12. Let $m \geqslant 1$ be an integer, let $h: A^{*} \rightarrow M$ be a surjective homomorphism onto an ordered monoid $M \in \mathbf{D A}$ satisfying $U_{m} \leqslant V_{m}$. There exists a positive integer $n$ such that $u \leqslant{ }_{m, n}^{\mathrm{FO} 2} v$ implies $h(u) \leqslant h(v)$ for all $u, v \in A^{*}$.

Proof. We proceed by induction on $m$. For the base case $m=1$ a result of Pin [20] shows that, for every $\leqslant$-order ideal $I$ of $M$, the set $h^{-1}(I)$ is a finite union of languages $A^{*} a_{1} \cdots A^{*} a_{k} A^{*}$ for some $k \geqslant 1$ and $a_{i} \in A$. Let $n$ be the maximum of all indices $k$ appearing in those unions when considering all order ideals $I \subseteq M$. If $u \leqslant \leqslant_{1, n}^{\mathrm{FO} 2} v$, then for all languages $P=A^{*} a_{1} \cdots A^{*} a_{k} A^{*}$ with $k \leqslant n$ we have that $v \in P$ implies $u \in P$. Moreover, the preimage $L$ of the order ideal generated by $h(v)$ is a finite union of languages $A^{*} a_{1} \cdots A^{*} a_{k} A^{*}$ with $k \leqslant n$. We have $v \in L$ and thus $u \in L$. This shows $h(u) \leqslant h(v)$.

In the following let $m \geqslant 2$ and fix some integer $\omega \geqslant 1$ such that $x^{\omega}$ is idempotent for all $x \in M$. We introduce a string rewriting system $\rightarrow$ on $A^{*}$ by letting $t \rightarrow s$ if $h(s)=h(t)$ or if $t=p v_{m-1} q$ and $s=p u_{m-1} q$ for $p, q \in A^{*}$, and $v_{1}=1$ and $u_{1}=z$, and for $i \geqslant 2$ we have

$$
v_{i}=\left(u_{i-1} x_{i}\right)^{\omega} v_{i-1}\left(y_{i} u_{i-1}\right)^{\omega}, \quad u_{i}=\left(u_{i-1} x_{i}\right)^{\omega} u_{i-1}\left(y_{i} u_{i-1}\right)^{\omega}
$$

for $x_{i}, y_{i}, z \in A^{*}$. Note that $t \rightarrow s$ implies $p^{\prime} t q^{\prime} \rightarrow p^{\prime} s q^{\prime}$ for all $p^{\prime}, q^{\prime} \in A^{*}$. Let $\stackrel{*}{\rightarrow}$ be the transitive closure of $\rightarrow$, i.e., let $t \stackrel{*}{\rightarrow} s$ if there exists a chain $t=w_{1} \rightarrow w_{2} \rightarrow \cdots \rightarrow w_{\ell}=s$ of rewriting steps for some $\ell \geqslant 1$ and $w_{i} \in A^{*}$. We claim that we can lift the rewriting steps of $t \stackrel{*}{\rightarrow} s$ to $M$ within certain contexts in an order respecting way.

Claim. Let $u, v, s, t \in A^{*}$ with $t \stackrel{*}{\rightarrow} s$. If both $h(u) \mathcal{R} h(u s)$ and $h(v) \mathcal{L} h(s v)$, then $h(u s v) \leqslant h(u t v)$.

The proof of the claim is by induction on the length of a minimal $\rightarrow$-chain from $t$ to $s$. The claim is trivial if $h(t)=h(s)$. Suppose $t \stackrel{*}{\rightarrow} t^{\prime} \rightarrow s$ and $t^{\prime}=p v_{m-1} q$ and $s=p u_{m-1} q$. Since $h(u) \mathcal{R} h(u s)$, there exists $x \in A^{*}$ such that $h(u)=h(u s x)$; and since $h(v) \mathcal{L} h(s v)$ there exists $y \in A^{*}$ such that $h(v)=h(y s v)$. Now $h(u)=h\left(u\left(p u_{m-1} q x\right)^{\omega}\right)$ and $h(v)=h\left(\left(y p u_{m-1} q\right)^{\omega} v\right)$. By letting $x_{m}=q x p$ and $y_{m}=q y p$, the identity $U_{m} \leqslant V_{m}$ of $M$ yields

$$
\begin{aligned}
h(u s v) & =h\left(u p\left(u_{m-1} x_{m}\right)^{\omega} u_{m-1}\left(y_{m} u_{m-1}\right)^{\omega} q v\right) \\
& \leqslant h\left(u p\left(u_{m-1} x_{m}\right)^{\omega} v_{m-1}\left(y_{m} u_{m-1}\right)^{\omega} q v\right)=h\left(u t^{\prime} v\right) .
\end{aligned}
$$


Observe that $\left(p u_{m-1} q x\right)^{\omega} p=p\left(u_{m-1} q x p\right)^{\omega}=p\left(u_{m-1} x_{m}\right)^{\omega}$. Note that $\operatorname{alph}\left(t^{\prime}\right) \subseteq \operatorname{alph}(s)$. Therefore, $h(u) \mathcal{R} h(u s)$ implies $h(u) \mathcal{R} h\left(u t^{\prime}\right)$, and symmetrically $h(v) \mathcal{L} h(s v)$ implies $h(v) \mathcal{L} h\left(t^{\prime} v\right)$. Induction yields $h\left(u t^{\prime} v\right) \leqslant h(u t v)$ and thus $h(u s v) \leqslant h(u t v)$. This completes the proof of the claim.

Let $t \sim s$ if $t \stackrel{*}{\rightarrow} s$ and $s \stackrel{*}{\rightarrow} t$. Let $M^{\prime}$ be the quotient $A^{*} / \sim$. The relation $\sim$ is a congruence on $A^{*}$ and $M^{\prime}$ is naturally equipped with a monoid structure. Let $h^{\prime}: A^{*} \rightarrow M^{\prime}$ be the canonical homomorphism mapping $u \in A^{*}$ to its equivalence class modulo $\sim$. The preorder $\stackrel{*}{\rightarrow}$ on $A^{*}$ induces a partial order on $M^{\prime}$ by letting $h^{\prime}(u) \leqslant h^{\prime}(v)$ whenever $v \stackrel{*}{\rightarrow} u$. Thus $M^{\prime}$ forms an ordered monoid. Moreover, $M^{\prime}$ is an unordered quotient of $M$ and, in particular, $M^{\prime}$ is finite and in $\mathbf{D A}$, and $x^{\omega}$ is idempotent for all $x \in M^{\prime}$.

By construction, $M^{\prime}$ satisfies the identity $U_{m-1} \leqslant V_{m-1}$ and induction yields an integer $n$ such that $u \leqslant{ }_{m-1, n}^{\mathrm{FO} 2} v$ implies $h^{\prime}(u) \leqslant h^{\prime}(v)$. We show that $u \leqslant{ }_{m, n^{\prime}}^{\mathrm{FO} 2} v$ implies $h(u) \leqslant h(v)$ for $n^{\prime}=n+2|M|$. Suppose $u \leqslant_{m, n^{\prime}}^{\mathrm{FO}^{2}} v$ and consider the factorizations $u=s_{0} a_{1} \cdots s_{\ell-1} a_{\ell} s_{\ell}$ and $v=t_{0} a_{1} \cdots t_{\ell-1} a_{\ell} t_{\ell}$ from Lemma 11. For all $i$ we have:

- $s_{i} \leqslant_{m-1, n}^{\mathrm{FO} 2} t_{i}$ and thus $t_{i} \stackrel{*}{\rightarrow} s_{i}$ by choice of $n$,

- $h\left(t_{0} a_{1} \cdots t_{i-1} a_{i}\right) \mathcal{R} h\left(t_{0} a_{1} \cdots t_{i-1} a_{i} s_{i}\right)$, and

- $h\left(a_{i+1} s_{i+1} \cdots a_{\ell} s_{\ell}\right) \mathcal{L} h\left(s_{i} a_{i+1} s_{i+1} \cdots a_{\ell} s_{\ell}\right)$.

For conciseness $t_{0} a_{1} \cdots t_{i-1} a_{i}$ is the empty word if $i=0$ and so is $a_{i+1} s_{i+1} \cdots a_{\ell} s_{\ell}$ if $i=\ell$. Applying the above claim repeatedly to substitute $s_{i}$ with $t_{i}$ for increasing $i \in\{0, \ldots, \ell\}$ yields the following chain of inequalities:

$$
\begin{aligned}
h(u) & =h\left(s_{0} a_{1} s_{1} \cdots s_{\ell-1} a_{\ell} s_{\ell}\right) \\
& \leqslant h\left(t_{0} a_{1} s_{1} \cdots s_{\ell-1} a_{\ell} s_{\ell}\right) \\
& \vdots \\
& \leqslant h\left(t_{0} a_{1} t_{1} \cdots t_{\ell-1} a_{\ell} s_{\ell}\right) \\
& \leqslant h\left(t_{0} a_{1} t_{1} \cdots t_{\ell-1} a_{\ell} t_{\ell}\right)=h(v) .
\end{aligned}
$$

Proof of Theorem 4. The implication (a) $\Rightarrow(\mathrm{b})$ is Lemma 6 , and $(\mathrm{b}) \Rightarrow(\mathrm{c})$ is Lemma 8 For the implication (c) $\Rightarrow$ (a), let $L \subseteq A^{*}$ be a language, let $h_{L}: A^{*} \rightarrow M_{L}$ be its syntactic homomorphism. Moreover, suppose that $M_{L}$ is in DA and satisfies $U_{m} \leqslant V_{m}$. The set $I=h_{L}(L)$ is an order ideal of $M_{L}$. Proposition 12 shows that there exists an integer $n$ such that $L=h_{L}^{-1}(I)$ is a union of $\leqslant_{m, n}^{\mathrm{FO} 2}$-order ideals. Up to equivalence, there are only finitely many formulas with quantifier depth $n$. Therefore, $\leqslant_{m, n}^{\mathrm{FO} 2}$-order ideals are $\Sigma_{m, n}^{2}$-definable.

Remark 1. The varieties $\mathbf{W}_{m}$ are similar to Straubing's characterization of $\mathrm{FO}_{m}^{2}$; the only difference is that the $\mathrm{FO}_{m}^{2}$ characterization starts with $\mathbf{J}$ instead of $\mathbf{J}^{+}$at level 1 (from where it also ascends by block products with $\mathbf{J}$ ). Intuitively, this is not surprising since the semantics of the innermost block in both fragments $\Sigma_{m}^{2}$ and $\mathrm{FO}_{m}^{2}$ is defined by the presence and absence of subwords, see Lemma 6 and [35, Theorem 4], respectively.

Remark 2. The identities for $\mathrm{FO}_{m}^{2}$ given by Krebs and Straubing [12, 35] are derived from a more general recursion scheme by Almeida and Weil [1, Theorem 8.8 (b)]. They 
follow a similar recursion scheme as $U_{m}$ and $V_{m}$. The main difference is that we use the idempotents $\left(U_{m-1} x_{m}\right)^{\omega}$ and $\left(y_{m} U_{m-1}\right)^{\omega}$ while the Almeida-Weil scheme would propose $\left(z x_{1} y_{1} \cdots x_{m-1} y_{m-1} x_{m}\right)^{\omega}$ and $\left(y_{m} z x_{1} y_{1} \cdots x_{m-1} y_{m-1}\right)^{\omega}$, respectively. One could easily adapt [12, 35] to our recursion scheme without any major changes in the proofs. The converse also works: One could use the Almeida-Weil recursion scheme in our setting; however, one would need to add some arguments in the proof of Proposition 12 (essentially, in the proof of the Claim one would have to additionally use the assumption $M \in \mathbf{D A}$ ). The crucial properties are that $U_{m-1} x_{m}$ and $z x_{1} y_{1} \cdots x_{m-1} y_{m-1} x_{m}$ (and $y_{m} U_{m-1}$ and $y_{m} z x_{1} y_{1} \cdots x_{m-1} y_{m-1}$, respectively) use the same variables, and that these variables form a superset of the variables occurring at level $m-1$. Our choice of identities was inspired by the identities in [17], and there we do not see whether or not the Almeida-Weil recursion scheme also works.

\section{Conclusion}

The fragments $\Sigma_{m}^{2}$ of $\mathrm{FO}^{2}[<]$ are defined by restricting the number of nested negations. They form the half levels of the alternation hierarchy $\mathrm{FO}_{m}^{2}$ in two-variable first-order logic, and we have $\Sigma_{m}^{2} \subseteq \mathrm{FO}_{m}^{2} \subseteq \Sigma_{m+1}^{2}$. It is known that the languages definable in $\mathrm{FO}_{m}^{2}$ form a strict hierarchy [43]. For every $m \geqslant 1$ we have given $\omega$-terms $U_{m}$ and $V_{m}$ such that a language $L$ is definable in $\Sigma_{m}^{2}$ if and only if its ordered syntactic monoid is in the variety DA and satisfies the identity $U_{m} \leqslant V_{m}$. Using this characterization one can decide whether a given regular language is definable in $\Sigma_{m}^{2}$. In particular, we have shown decidability for every level of an infinite hierarchy. Note that there is no immediate connection between the decidability of $\mathrm{FO}_{m}^{2}$ and the decidability of $\Sigma_{m}^{2}$.

The block product principle is an important tool in the proof of the direction from $\Sigma_{m}^{2}$ to the identities. In order to be able to apply this tool, we first extended block products to the case where the left factor is an ordered monoid and then stated the block product principle in this context. For further extending the block product $M \square N$ to the case where both $M$ and $N$ are ordered, the work of Pin and Weil on the wreath product principle [23] for ordered monoids suggests to consider the monotone functions in $N \times N \rightarrow M$ instead of $M^{N \times N}$. This leads to ordered alphabets when stating the block product principle. However, one implication in the block product principle fails for ordered alphabets as the universal property does not hold in this setting.

It would be interesting to see whether the proof scheme in [12] could also be used for proving our characterization of $\Sigma_{m}^{2}$.

\section{References}

[1] J. Almeida and P. Weil. Profinite categories and semidirect products. J. Pure Appl. Algebra, 123(1-3):1-50, 1998.

[2] J. R. Büchi. Weak second-order arithmetic and finite automata. Z. Math. Logik Grundlagen Math., 6:66-92, 1960. 
[3] R. S. Cohen and J. A. Brzozowski. Dot-depth of star-free events. J. Comput. Syst. Sci., $5(1): 1-16,1971$.

[4] V. Diekert, P. Gastin, and M. Kufleitner. A survey on small fragments of first-order logic over finite words. Int. J. Found. Comput. Sci., 19(3):513-548, 2008.

[5] H.-D. Ebbinghaus and J. Flum. Finite Model Theory. Perspectives in Mathematical Logic. Springer, 1995.

[6] S. Eilenberg. Automata, Languages, and Machines, volume B. Academic Press, New York and London, 1976.

[7] C. C. Elgot. Decision problems of finite automata design and related arithmetics. Trans. Amer. Math. Soc., 98:21-51, 1961.

[8] L. Fleischer, M. Kufleitner and A. Lauser. Block products and nesting negations in $\mathrm{FO}^{2}$. In CSR 2014, Proceedings, volume 8476 of LNCS, pp. 176-189. Springer, 2014.

[9] Ch. Glaßer and H. Schmitz. Languages of dot-depth 3/2. Theory of Computing Systems, 42(2):256-286, 2008.

[10] J. A. W. Kamp. Tense Logic and the Theory of Linear Order. PhD thesis, University of California, 1968.

[11] R. Knast. A semigroup characterization of dot-depth one languages. RAIRO, Inf. Théor., 17(4):321-330, 1983.

[12] A. Krebs and H. Straubing. An effective characterization of the alternation hierarchy in two-variable logic. In FSTTCS 2012, Proceedings, volume 18 of LIPIcs, pages 86-98. Dagstuhl Publishing, 2012.

[13] M. Kufleitner and A. Lauser. Languages of dot-depth one over infinite words. In LICS 2011, Proceedings, pages 23-32. IEEE Computer Society, 2011.

[14] M. Kufleitner and A. Lauser. Around dot-depth one. Int. J. Found. Comput. Sci., 23(6):13231339, 2012.

[15] M. Kufleitner and A. Lauser. The join levels of the Trotter-Weil hierarchy are decidable. In MFCS 2012, Proceedings, volume 7464 of LNCS, pages 603-614. Springer, 2012.

[16] M. Kufleitner and A. Lauser. The join of the varieties of R-trivial and L-trivial monoids via combinatorics on words. Discrete Math. \& Theor. Comput. Sci., 14(1):141-146, 2012.

[17] M. Kufleitner and A. Lauser. Quantifier alternation in two-variable first-order logic with successor is decidable. In STACS 2013, Proceedings, volume 20 of LIPIcs, pages 305-316. Dagstuhl Publishing, 2013.

[18] M. Kufleitner and P. Weil. The $\mathrm{FO}^{2}$ alternation hierarchy is decidable. In CSL 2012, Proceedings, volume 16 of LIPIcs, pages 426-439. Dagstuhl Publishing, 2012.

[19] R. McNaughton and S. Papert. Counter-Free Automata. The MIT Press, 1971.

[20] J.-É. Pin. A variety theorem without complementation. In Russian Mathematics (Iz. VUZ), volume 39, pages 80-90, 1995.

[21] J.-É. Pin and P. Weil. Polynomial closure and unambiguous product. Theory Comput. Syst., 30(4):383-422, 1997.

[22] J.-É. Pin and P. Weil. Semidirect products of ordered semigroups. Commun. Algebra, 30(1):149-169, 2002.

[23] J.-É. Pin and P. Weil. The wreath product principle for ordered semigroups. Commun. Algebra, 30(12):5677-5713, 2002.

[24] Th. Place and M. Zeitoun. Going higher in the first-order quantifier alternation hierarchy on words. In ICALP 2014, Proceedings, volume 8573 of LNCS, pp. 342-353. Springer, 2014. 
[25] J. Rhodes and B. Tilson. The kernel of monoid morphisms. J. Pure Appl. Algebra, 62(3):227268, 1989.

[26] J. Rhodes and P. Weil. Decomposition techniques for finite semigroups, using categories II. J. Pure Appl. Algebra, 62(3):285-312, 1989.

[27] M. P. Schützenberger. On finite monoids having only trivial subgroups. Inf. Control, 8:190-194, 1965.

[28] Th. Schwentick, D. Thérien, and H. Vollmer. Partially-ordered two-way automata: A new characterization of DA. In DLT 2001, Proceedings, volume 2295 of LNCS, pages 239-250. Springer, 2002.

[29] I. Simon. Piecewise testable events. In Autom. Theor. Form. Lang., 2nd GI Conf., volume 33 of $L N C S$, pages 214-222. Springer, 1975.

[30] H. Straubing. Families of recognizable sets corresponding to certain varieties of finite monoids. J. Pure Appl. Algebra, 15(3):305-318, 1979.

[31] H. Straubing. Aperiodic homomorphisms and the concatenation product of recognizable sets. J. Pure Appl. Algebra, 15(3):319-327, 1979.

[32] H. Straubing. A generalization of the Schützenberger product of finite monoids. Theor. Comput. Sci., 13:137-150, 1981.

[33] H. Straubing. Finite semigroup varieties of the form $\mathbf{V} * \mathbf{D}$. J. Pure Appl. Algebra, 36(1):53-94, 1985.

[34] H. Straubing. Finite Automata, Formal Logic, and Circuit Complexity. Birkhäuser, 1994.

[35] H. Straubing. Algebraic characterization of the alternation hierarchy in $\mathrm{FO}^{2}[<]$ on finite words. In CSL 2011, Proceedings, volume 12 of LIPIcs, pages 525-537. Dagstuhl Publishing, 2011.

[36] P. Tesson and D. Thérien. Diamonds are forever: The variety DA. In Semigroups, Algorithms, Automata and Languages 2001, Proceedings, pages 475-500. World Scientific, 2002.

[37] D. Thérien. Classification of finite monoids: The language approach. Theor. Comput. Sci., 14(2):195-208, 1981.

[38] D. Thérien. Two-sided wreath product of categories. J. Pure Appl. Algebra, 74(3):307-315, 1991.

[39] D. Thérien and Th. Wilke. Over words, two variables are as powerful as one quantifier alternation. In STOC 1998, Proceedings, pages 234-240. ACM Press, 1998.

[40] W. Thomas. Classifying regular events in symbolic logic. J. Comput. Syst. Sci., 25:360-376, 1982.

[41] B. A. Trakhtenbrot. Finite automata and logic of monadic predicates. Dokl. Akad. Nauk SSSR, 140:326-329, 1961. in Russian.

[42] P. Weil. Closure of varieties of languages under products with counter. J. Comput. Syst. Sci., 45(3):316-339, 1992.

[43] $\mathrm{Ph}$. Weis and N. Immerman. Structure theorem and strict alternation hierarchy for $\mathrm{FO}^{2}$ on words. Log. Methods Comput. Sci., 5(3):1-23, 2009. 